\title{
Crisis, migración y estructura de empleo en Baja California
}

\section{Crisis, migration and employment structure in Baja California}

\author{
Alejandro Mungaray, ${ }^{*}$ Antonio Escamilla, ${ }^{*}$ Natanael Ramírez* \\ y José G. Aguilar
}

\section{Resumen}

En este documento se revisa la dinámica económica y demográfica en Baja California que se ha caracterizado por ser distinta a la del resto del país, debido a los constantes flujos poblacionales que llegan a la región. Mediante un análisis deductivo se muestra que debido a sus fuertes vínculos con el exterior, durante la crisis de 2009 la economía de la entidad se vio afectada principalmente en el sector manufacturero y en la construcción. Sin embargo, y contrario a lo ocurrido en las demás entidades de la región, se dieron importantes efectos compensatorios en los sectores comercial y primario. Estos mismos vínculos están impulsando una prudente recuperación del empleo, principalmente en las Mipymes (Micro, Pequeñas y Medianas Empresas), con sus respectivos impactos en el resto de la economía.

Palabras clave: desarrollo económico 018, empleo J61, migración regional R23, estructura industrial L11, economía regional R11.

Recibido el 20 de noviembre de 2013.

Aprobado el 12 de diciembre de 2013.

\begin{abstract}
This document analyzes the economic and demographic dynamics in Baja California, which has been characterized for being different from the rest of the country, due to the constant population flows arriving at the region. Through a deductive analysis it shows that, due to its strong links with the California economy, during the crisis in 2009 the state economy was affected mainly in the manufacturing and construction sectors. However and contrary to what happened in other states in the country, there were important compensatory effects on the primary and commercial sectors. These same links are propelling a careful employment recovery in the small business mainly, with its respective impacts on the rest of the economy.

Keywords: economic development 018, employment J61, regional migration R23, industrial structure L11, regional economy R11.
\end{abstract}

* Profesores de la Facultad de Economía y Relaciones Internacionales de la Universidad Autónoma de Baja California. Correos electrónicos: mungaray@uabc.edu.mx; escamillaa@uabc.edu.mx; natanael@uabc.edu.mx y gaba@uabc.edu.mx 


\section{Introducción}

Baja California se ha caracterizado por una dinámica demográfica y económica distinta a la del resto del país, debido a los constantes flujos poblacionales que llegan a la región al percibirla como una zona de oportunidad para sus habitantes. Al transitar por una difícil coyuntura económica mundial no vista desde 1929, los flujos migratorios ilustran el creciente atractivo del Estado como una alternativa para alcanzar un mejor nivel de vida. Igualmente, la capacidad emprendedora en Baja California se observa en el fuerte aumento de la población ocupada en los micronegocios en la entidad.

$\mathrm{Al}$ evaluar los impactos diferenciados de la crisis de 2009 y la importancia de la estructura empresarial en el empleo en Baja California, se encuentra que debido a sus fuertes vínculos con el exterior, durante la crisis de 2009 la economía de la entidad se vio afectada principalmente en el sector manufacturero y en la construcción. Sin embargo, y contrario a lo ocurrido en las demás entidades de la región, se dieron importantes efectos compensatorios en los sectores comercial y primario que se vieron beneficiados por la depreciación del tipo de cambio. Mediante un método deductivo se construye la relación entre la crisis global y la reestructuración de los niveles tecnológicos de las actividades de maquila: entre éstos y la respuesta que las Mipymes (Micro, Pequeñas y Medianas Empresas) dan para absorver el empleo que dejan de generar las maquiladoras y la nueva demanda de empleo que genera la migración proveniente del sur del país.

Para tal fin, el documento se ordena de la manera siguiente: en el segundo apartado se presenta un análisis de la evolución de la economía mundial y también se muestra cómo la relación entre Baja California y California genera un impacto en la actividad económica de esta macrorregión transfronteriza. En el tercer apartado se realiza una revisión de la dinámica poblacional, considerando el crecimiento de la migración como elemento fundamental para el aumento de la población económicamente activa.

En el cuarto apartado se expone la estructura económica y de la ocupación en el estado. En el quinto, se resalta la importancia de la industria maquiladora en la organización industrial y se enfatiza el papel de las 
microempresas y las pequeñas empresas como la principal fuente de creación de empleo y como efecto compensatorio ante la pérdida del empleo de la gran empresa. Finalmente, en el sexto apartado se presentan las conclusiones con énfasis en algunas perspectivas que resaltan la necesidad de fortalecer, en la política de desarrollo empresarial, el énfasis a la empresarialidad local, con base en el impulso a la micro, pequeña y mediana empresas.

\section{Tendencias macroecónomicas y desarrollo regional}

La situación económica mundial a finales de 2008 empezó a mostrar síntomas de la difícil situación que se presentaría en el año 2009 como consecuencia del incremento de la cartera vencida asociada a la venta de casas en Estados Unidos. La crisis hipotecaria primero dio lugar al aumento de la incertidumbre y posteriormente a una crisis financiera a escala mundial que afectó de manera severa la confianza del consumidor y desencadenó una drástica caída en el comercio y la producción alrededor del mundo. El rápido deterioro de las condiciones económicas ocasionó que en julio de 2009 los organismos internacionales revisaran a la baja sus proyecciones realizadas en octubre de 2008, sobre la evolución de la economía mundial. (cuadro 1).

En las estimaciones de enero de 2009 se pronosticaba un crecimiento de $0.5 \%$ para la economía mundial en su conjunto, y en 2009 ya se registraba una disminución de $0.6 \%$. Esta situación fue generalizada a nivel de países. En Estados Unidos el deterioro de las expectativas pasó de una reducción de su Producto Interno Bruto (РIB) de $-1.6 \%$, a una de $-2.6 \%$. En el caso de México y Brasil, principales economías latinoamericanas, se pasó de un PIB de $-0.3 \%$ y $1.8 \%$ a una disminución de $-6.1 \%$ y $-0.6 \%$, respectivamente.

Los impactos de la crisis no han sido homogéneos. Más bien se observan diferenciados en el tiempo, sectores y regiones. En principio, los efectos impactaron las zonas con un mayor desarrollo financiero y posteriormente a las menos desarrolladas. A nivel de sectores pasó de la industria de la construcción al sector industrial y después al comercio y los servicios (Mendoza, 2010). 


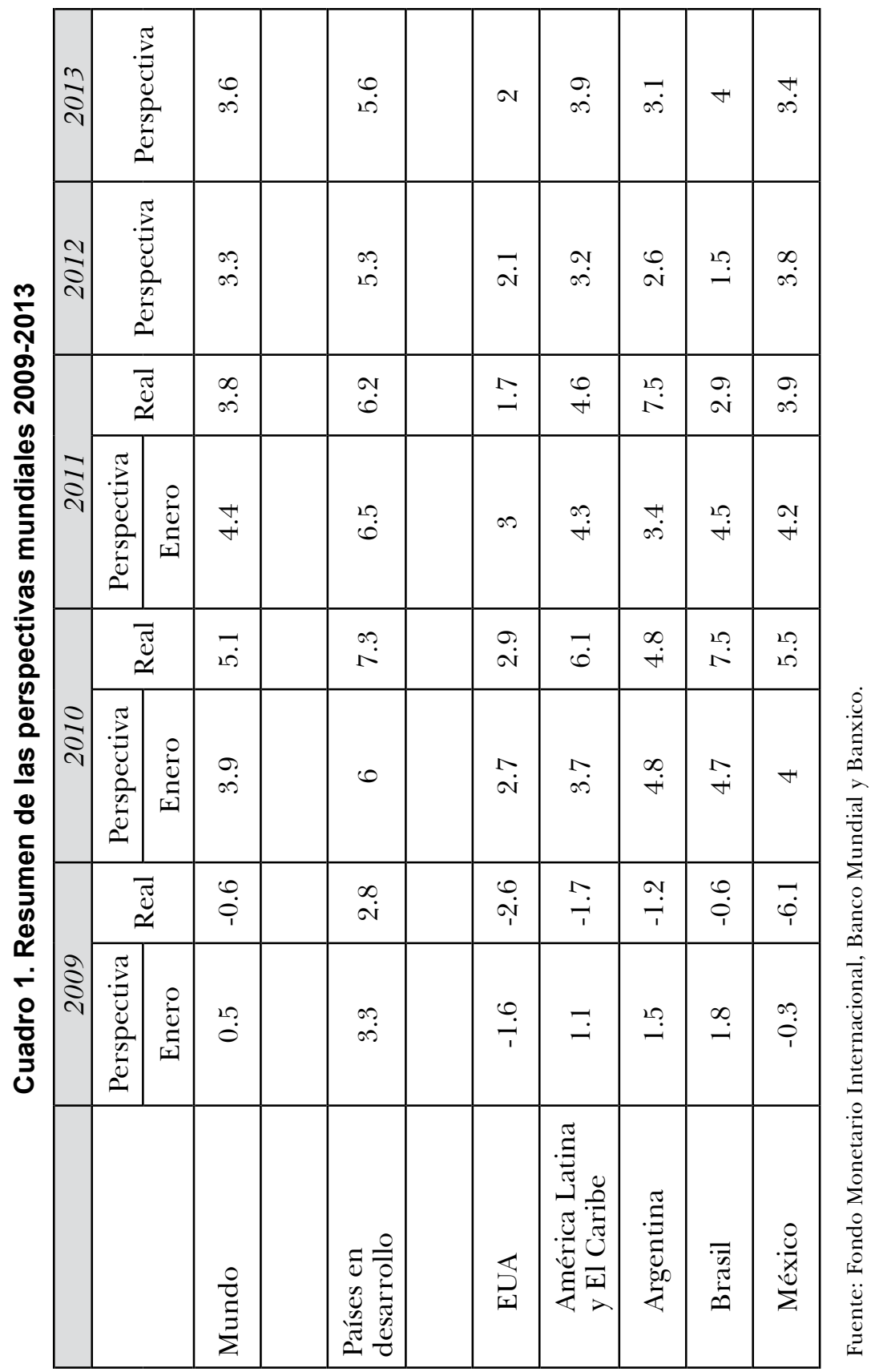


Los gobiernos nacionales reaccionaron con la implementación de medidas anticíclicas ante la evidencia del agravamiento de la crisis económica y financiera internacional, destacando la intensa actividad de los bancos centrales para proveer de liquidez a los sistemas financieros locales, en moneda nacional y extranjera, a fin de permitir el normal funcionamiento de los mercados de crédito. También se otorgaron incentivos de política fiscal como exenciones en el pago de impuestos y se dio un incremento en el gasto público con el objetivo de incentivar la recuperación de los mercados. En el caso de México se observó una caída en las exportaciones de productos y servicios a Estados Unidos; una fuerte disminución de la inversión extranjera, debido a la reducción del flujo de inversión a escala mundial; una caída de los ingresos públicos por la disminución de impuestos; una disminución en el envío de remesas por la caída del empleo de los mexicanos residentes en Estados Unidos; y una reducción de los ingresos turísticos, situación agravada con la contingencia sanitaria por el brote de influenza AH1N1.

A escala regional la intensa relación entre Baja California y California, en los intensos flujos de comercio tanto como de personas, generó un impacto determinante en la actividad económica de esta macrorregión transfronteriza, donde Baja California participa con $51 \%$ del comercio exterior que tiene California con México. Esta situación ubica a Baja California como el tercer socio comercial de California, sólo detrás de México y Canadá y en un nivel similar al de Japón. A ello contribuye el hecho de que Baja California es la entidad con la economía más abierta del país, con un comercio exterior que representa 2.8 veces el tamaño de su PIB según el Instituto Mexicano para la Competitividad (Imco, 2008).

De acuerdo con el Bureau of Transportation and Statistics de Estados Unidos, las exportaciones de California a Baja California mantuvieron una tendencia creciente al pasar de 4942 a 12137 millones de dólares (MDD) entre 1995 y 2008, en que se observa el mayor nivel de intercambio comercial. Sin embargo, a consecuencia de la fuerte disminución de la actividad industrial en la región transfronteriza, durante 2009 se observa una disminución de $13 \%$ en los flujos de bienes de California a la entidad; y en 2011 una recuperación de 11676 mDD, con lo cual la disminución se reduce a 3.8 por ciento (figura 1 ).

Por su parte, no obstante la disminución propiciada por los tiempos de espera en los cruces fronterizos y la disminución en la actividad económica 
Figura 1. Exportaciones de California a Baja California, 1995-2011 (Millones de dólares)

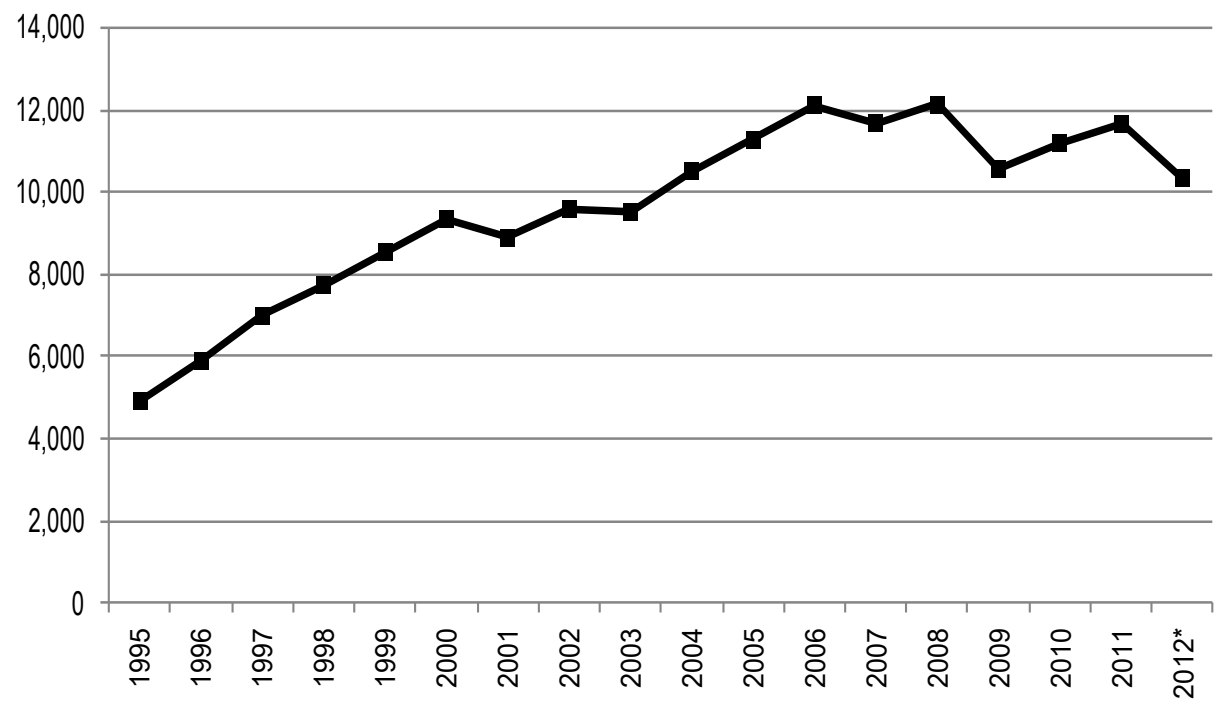

Fuente: Bureau of Transportation an Statistics * De enero a octubre.

que reduce los cruces de trabajadores transfronterizos, el flujo de personas y vehículos aún mantiene a la región como la más dinámica de la frontera norte de México y a escala mundial. De hecho, la región de San Diego y Tijuana es la zona urbana y económica fronteriza más grande a lo largo de toda la frontera México-Estados Unidos, con una población combinada de más de cuatro millones de personas, la cual se prevé aumentará a más de 5.5 millones en 2020 (San Diego Regional Planning Agency, 2008, y Conapo, 2010).

\section{Migración y cambio demográfico}

La configuración original del mercado fronterizo de Baja California se caracterizó por la ausencia de la industria nacional y la fuerte presencia de hábitos norteamericanos de consumo. Ello ha hecho arduo el desarrollo 
de un mercado interno fuerte y competitivo que premie al consumidor y garantice al productor las condiciones necesarias para desarrollar dicho mercado. En consecuencia, el desarrollo económico fue alentado con un régimen de zona libre que permitió la formación de un fuerte sector empresarial en todas las actividades económicas, principalmente en el comercio y los servicios, condicionado por la vocación histórica asociada al origen de las ciudades fronterizas que surgieron fiscalmente apoyadas como puertos de comercio y abasto de los estados y las regiones fronterizas (Mungaray y Samaniego, 2006) (cuadro 2).

El cambio demográfico en Baja California ha sido determinado por el flujo migratorio relacionado con los nuevos patrones de localización industrial, comercial y de servicios derivados de la reestructuración económica regional del país (Estrella, 1998). Entre 1940 y 1960 la población de Baja California creció a un ritmo anual de $10 \%$. Por su cercanía con el mercado laboral de California, sus ciudades se convirtieron en un importante destino de la migración interna. En espera de una oportunidad remunerada en el Programa Bracero acordado entre México y Estados Unidos durante

Cuadro 2. Evolución de la población, 1960-2013

\begin{tabular}{|l|r|r|r|r|r|r|}
\hline \multicolumn{1}{|c|}{ Periodo } & \multicolumn{1}{|c|}{$\begin{array}{c}\text { Baja } \\
\text { California }\end{array}$} & \multicolumn{1}{c|}{$T C$} & \multicolumn{1}{c|}{ Frontera Norte } & \multicolumn{1}{c|}{$T C$} & \multicolumn{1}{c|}{ Nacional } & $T C$ \\
\hline 1960 & 520165 & 8.3 & 5538100 & 3.9 & 34923129 & 3.0 \\
\hline 1970 & 870421 & 5.2 & 7848169 & 3.5 & 48225238 & 3.2 \\
\hline 1980 & 1177886 & 3.0 & 10691887 & 3.1 & 66846833 & 3.3 \\
\hline 1990 & 1660855 & 3.4 & 13246991 & 2.1 & 81249645 & 2.0 \\
\hline 2000 & 2487367 & 4.0 & 16642676 & 2.3 & 97483412 & 1.8 \\
\hline 2010 & 3155070 & 2.7 & 19894418 & 1.8 & 112336538 & 1.4 \\
\hline 2013 & 3375913 & 2.3 & 21130681 & 2.1 & 118129240 & 1.8 \\
\hline $1960-2013$ & 2855748 & 3.3 & 15592581 & 2.0 & 83206111 & 1.7 \\
\hline
\end{tabular}

Fuente: INEgi y Consejo Nacional de Población. 
1940 y 1964, mucha gente se concentró en Tijuana y Mexicali, presionando con su presencia para que los servicios públicos fueran permanentemente suficientes. Cuando el programa se dio por concluido, el desempleo agravó la situación social. La puesta en marcha del Programa de Industrialización Fronteriza, en 1965, fue un importante paliativo. No obstante, la dinámica poblacional continúa presionando la infraestructura estatal debido a las crecientes oportunidades de empleo que brinda la agricultura de exportación en gran escala que se desarrolla en los valles; del fuerte sector comercial y de servicios que con los años se ha formado para atender a los visitantes del sur de California que se internan con múltiples motivos en la frontera mexicana; y del dinamismo industrial que ha caracterizado los últimos tiempos del crecimiento económico bajacaliforniano.

Ello no significa que la esperanza de trabajar en Estados Unidos haya disminuido como motivo migratorio al estado. Sin embargo, si bien es notable el descenso en el crecimiento de la población entre 2000 y 2010 en relación con el resto de la frontera y el país, es indicio de que su ritmo ha empezado a estabilizarse, pues pasó de $4 \%$ a $2.4 \%$ anual. Esto resulta lógico si se piensa que la economía estatal, por la vocación exportadora desarrollada desde la década de 1960, ha crecido en términos reales, mientras que la del país ha enfrentado severos ajustes que han desalentado muchas actividades económicas tradicionales y rurales, estimulando a su vez la migración hacia un estado que se ha convertido en un demandante permanente de fuerza de trabajo por haberse convertido en un centro de producción para el mercado mundial, con el consecuente incremento de la población económicamente activa (PEA) (cuadro 3).

El incremento sostenido en la PEA de Baja California en $56 \%$ durante los últimos 15 años, es superior al 39\% registrado en la frontera norte en su conjunto y al $33 \%$ observado a escala nacional. Más aún, se puede notar que posterior a la crisis de 2009, la información censal de 2010 muestra cómo dentro del total de la población migrante a la frontera norte de México, la que migró a Baja California, si bien disminuyó su peso de $31 \%$ a $26 \%$ entre 2005 y 2010, se mantiene aún por encima de Nuevo León, que creció de $18 \%$ a $23 \%$. Esto corrobora el hecho de que la movilidad de la población hacia las regiones con mejores expectativas de mejora en la ocupación y el nivel de vida seguirá siendo una constante que estará presionando a Baja California a buscar alternativas permanentes de crecimiento (cuadro 4). 
Cuadro 3. Población económicamente activa, 1998-2012

\begin{tabular}{|c|c|c|c|c|c|c|}
\hline Periodo & $\begin{array}{c}\text { Baja } \\
\text { California }\end{array}$ & $T C$ & Frontera norte & $T C$ & Nacional & $T C$ \\
\hline 1998 & 924304 & & 6675297 & & 38244313 & \\
\hline 1999 & 965453 & 4.45 & 6656456 & -0.28 & 38234069 & -0.03 \\
\hline 2000 & 1017243 & 5.36 & 6924058 & 4.02 & 39325442 & 2.85 \\
\hline 2001 & 1048831 & 3.11 & 6972366 & 0.70 & 39800112 & 1.21 \\
\hline 2002 & 1060351 & 1.10 & 7074242 & 1.46 & 40094511 & 0.74 \\
\hline 2003 & 1088575 & 2.66 & 7269114 & 2.75 & 41280968 & 2.96 \\
\hline 2004 & 1123860 & 3.24 & 7524823 & 3.52 & 41970528 & 1.67 \\
\hline 2005 & 1200414 & 6.81 & 7781167 & 3.41 & 43232383 & 3.01 \\
\hline 2006 & 1215058 & 1.22 & 8194901 & 5.32 & 45807786 & 5.96 \\
\hline 2007 & 1300491 & 7.03 & 8512493 & 3.88 & 47018401 & 2.64 \\
\hline 2008 & 1327792 & 2.10 & 8491053 & -0.25 & 46561352 & -0.97 \\
\hline 2009 & 1388670 & 4.58 & 8799164 & 3.63 & 48482106 & 4.13 \\
\hline 2010 & 1391841 & 0.23 & 8624254 & -1.99 & 47900977 & -1.20 \\
\hline 2011 & 1493263 & 7.29 & 9150866 & 6.11 & 50273465 & 4.95 \\
\hline 2012 & 1502071 & 0.59 & 9262961 & 1.22 & 50693306 & 0.84 \\
\hline 1999-2012 & 536618 & 55.58 & 2606505 & 39.16 & 12459237 & 32.59 \\
\hline
\end{tabular}

Fuente: Encuesta Nacional de Educación y Empleo, INEGI. 
Cuadro 4. Población inmigrante de la frontera norte de 5 y más con residencia cinco años antes en 2000, 2005 y 2010

\begin{tabular}{|c|c|c|c|c|c|c|}
\hline Entidad federativa & 2000 & $\%$ & 2005 & $\%$ & 2010 & $\%$ \\
\hline Baja California & 229547 & 28.3 & 170169 & 31.3 & 154029 & 26.0 \\
\hline Coahuila & 72981 & 9.0 & 45539 & 8.4 & 61636 & 10.4 \\
\hline Coahuila & 138616 & 17.1 & 67483 & 12.4 & 58334 & 9.8 \\
\hline Nuevo León & 128902 & 15.9 & 96326 & 17.7 & 133657 & 22.6 \\
\hline Sonora & 77072 & 9.5 & 51024 & 9.4 & 78545 & 13.3 \\
\hline Tamaulipas & 164697 & 20.3 & 113953 & 20.9 & 106410 & 18.0 \\
\hline
\end{tabular}

Fuente: Censo de Población, INEgI.

\section{Estructura económica y ocupación}

Si bien la vocación como economía de servicios en mucho explica el origen de las ciudades de Baja California, a partir de 1970 el Programa de Comercialización Fronteriza estimula la educación social hacia el consumo de bienes de origen nacional y propicia una fuerte conversión hacia el comercio y los servicios de las actividades económicas en Mexicali y Ensenada (Mungaray y Moctezuma, 1988). El largo periodo de ajuste cambiario entre 1976 y 1994 terminó por modificar el tradicional patrón de consumo bajacaliforniano (Mungaray y Ocegueda, 1995), pues ante los intensos procesos devaluatorios, la tradicional economía estatal orientada al comercio y los servicios empezó a perder competitividad y fueron las actividades industriales de maquila las nuevas ganadoras, pues el impacto sobre los costos de los insumos nacionales, principalmente de fuerza de trabajo, permitió el crecimiento de las exportaciones industriales y agroindustriales del estado (cuadro 5). 
Cuadro 5. Estructura sectorial del empleo en Baja California, 1960-2012

\begin{tabular}{|c|c|c|c|c|}
\hline Año/Sector & Total & Primario & Industrial & Servicios \\
\hline 1960 & 167436 & 66042 & 30652 & 70742 \\
\hline$\%$ & 100 & 39.4 & 18.3 & 42.3 \\
\hline 1970 & 202241 & 49440 & 37070 & 115731 \\
\hline$\%$ & 100 & 24.4 & 18.3 & 57.2 \\
\hline 1980 & 403279 & 38180 & 81648 & 283451 \\
\hline$\%$ & 100 & 9.5 & 20.2 & 70.3 \\
\hline 1990 & 565471 & 58584 & 179527 & 327360 \\
\hline$\%$ & 100 & 10.4 & 31.7 & 57.9 \\
\hline 2000 & 999340 & 48072 & 344076 & 607192 \\
\hline$\%$ & 100 & 4.8 & 34.4 & 60.8 \\
\hline 2010 & 1232324 & 87720 & 333594 & 811010 \\
\hline$\%$ & 100 & 7.1 & 27.1 & 65.8 \\
\hline 2012 & 1331359 & 95014 & 354846 & 881499 \\
\hline$\%$ & 100 & 7.1 & 26.7 & 66.2 \\
\hline $1960-1970$ & 1.9 & -2.9 & 1.9 & 5.0 \\
\hline $1970-1980$ & 7.1 & -2.6 & 8.2 & 9.4 \\
\hline 1980-1990 & 3.4 & 4.4 & 8.2 & 1.5 \\
\hline $1990-2000$ & 5.9 & -2.0 & 6.7 & 6.4 \\
\hline $2000-2010$ & 2.1 & 6.2 & -0.3 & 2.9 \\
\hline $2010-2012$ & 3.1 & 3.2 & 2.5 & 3.4 \\
\hline
\end{tabular}

Fuente: Censos generales de población, Encuesta Nacional de Empleo y Encuesta Nacional de Ocupación y Empleo, INEGI. 
Quizá la tendencia más significativa de la economía es la baja sostenida en la participación en el empleo del sector primario y el crecimiento permanente de la participación del empleo comercial y de servicios e industrial, si bien una buena parte del rápido descenso de la población involucrada en actividades agrícolas se debe al incremento de la mecanización y productividad del campo. La información disponible indica que si bien el empleo industrial ha crecido de manera sostenida entre $1960 \mathrm{y}$ 2000, para 2012, aunque en términos absolutos siguió creciendo, su peso relativo disminuyó. Su crecimiento entre 1960 y 1970 sin duda se debe a la puesta en marcha del Programa de Industrialización Fronteriza en 1965 y a la inmediata presencia de plantas maquiladoras que ejercieron fuerte demanda sobre el mercado de trabajo. A mediados de la década de 1970, y debido a un fuerte ambiente recesivo de la economía norteamericana y mundial, muchas plantas cerraron sus puertas, desempleando a gran cantidad de gente. Por el contrario, el empleo en el comercio y los servicios creció aceleradamente durante la misma década, debido al fuerte impacto que sobre la generación de negocios comerciales tuvieran los programas Nacional Fronterizo y de Comercialización Fronteriza; pero también porque las políticas de sobrevaluación de la época estimularon fuertemente las actividades económicas de este sector en las ciudades bajacalifornianas.

La capacidad de la economía del sur de Estados Unidos para satisfacer las necesidades del mercado fronterizo y las dificultades de la producción nacional para competir en precio y calidad, son factores importantes para explicar el surgimiento de un empresariado comercial sumamente ágil y agresivo, pues aunque su prosperidad en el comercio de una gran cantidad de bienes y servicios extranjeros se asocia a la sobrevaluación, a partir de la devaluación de 1976 empezó a mostrar su flexibilidad para ofertar bienes nacionales o extranjeros según fuera el caso. Estas circunstancias permitieron que el sector comercial y de servicios se caracterizara por ser el más dinámico generador de empleos en la economía fronteriza, pasando entre 1960 y 1980 del $42.3 \%$ al $70.3 \%$ de los mismos; y a pesar de una considerable disminución al 58\% en 1990 por el gran incremento del peso del empleo manufacturero, para 2012 muestra una notable participación de $62.3 \%$, debido al crecimiento de las ventas al menudeo y la ampliación de la disponibilidad de bienes y servicios. Sin duda en esto influye el diferencial de $5 \%$ en el iva fronterizo respecto al del país y la entrada en 
vigor del Decreto de Competitividad y Reducción Arancelaria de la Zona Económica Fronteriza, en enero de 2012, que disminuye la Tarifa del Impuesto General de Importación de entre 10\% y 35\% hasta 5\% para 354 fracciones arancelarias y hasta $0 \%$ para 50 (DOF, 2012).

La actividad económica de Baja California, desarrollada en un ambiente de apertura comercial, pasó de ser predominantemente agrícola en 1960 a evidentemente urbana, orientada fuertemente por el comercio y los servicios, con una creciente presencia de la actividad industrial hacia 1990. A su vez, ésta es dirigida por la industria maquiladora y por algunos segmentos no maquiladores con alta inversión extranjera (Escamilla y Ocegueda, 2011). De 2003 a 2011 la producción manufacturera tuvo un crecimiento promedio anual de $3.3 \%$ y entre 2003 y 2006 aumentó su participación en el PIB de $21.2 \%$ a 23.0\%. Posteriormente, y tras la desaceleración de la economía estadunidense y el ascenso de otros sectores, la participación de este sector se mantiene en el orden del $22.7 \%$ hasta 2011. No obstante, otros subsectores de la manufactura crecen a tasas cercanas o incluso mayores al $5 \%$ promedio anual durante el periodo en cuestión, tales como industria del papel, impresión e industrias conexas (5.8\%), maquinaria y equipo $(5.6 \%)$ y otras industrias manufactureras (5.5\%).

El liderazgo del subsector 333-336 dentro de las actividades manufactureras del estado, se ha consolidado por la presencia de empresas de clase mundial dentro de la industria maquiladora, principalmente del giro aeroespacial. Esto se debe a que la entidad se ha constituido en un espacio ideal para distribuir las fases de procesos productivos, con la posibilidad de armonizar al mismo tiempo producción, proveedores y mercado.

El comercio y los servicios de transporte, correo y almacenamiento presentan durante el periodo 2003-2011 una tasa anual de crecimiento de $3.1 \%$ y $2.5 \%$ respectivamente. Esto les ha permitido incrementar su participación dentro del PIB estatal de $16.3 \%$ a $17.1 \%$ entre 2003 y 2011 para los sectores 43 y 46 , y de $6.7 \%$ a $6.8 \%$ para los sectores 48 y 49 . El sector que muestra los incrementos más pronunciados en su participación dentro del PIB estatal es maquinaria y equipo, debido a su estrecha relación con las actividades de la industria maquiladora y de comercio exterior que ha venido intensificándose (cuadro 6).

En este contexto estatal, se debe reconocer que los municipios de Baja California son heterogéneos, pues las vocaciones económicas propias de 


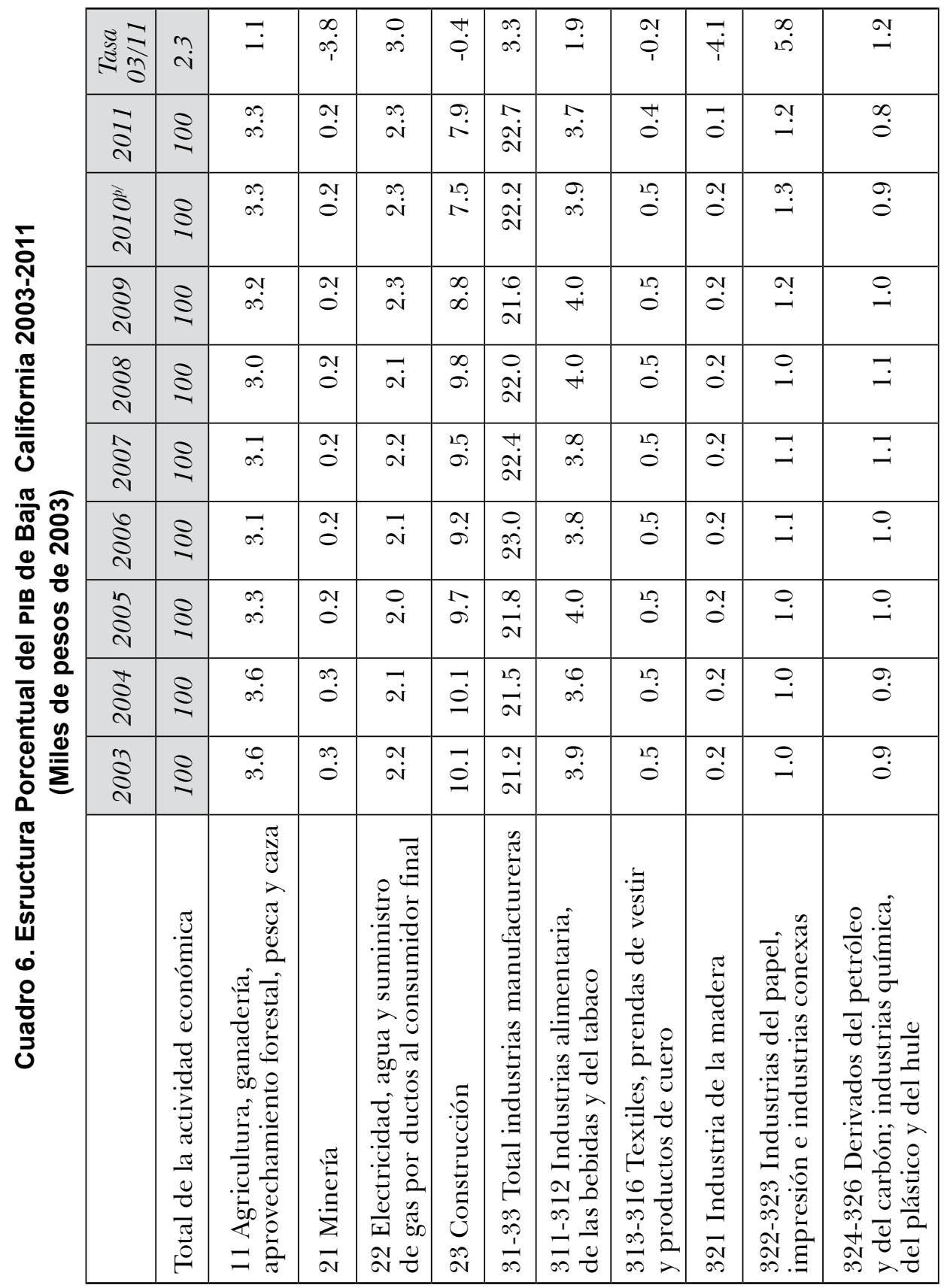




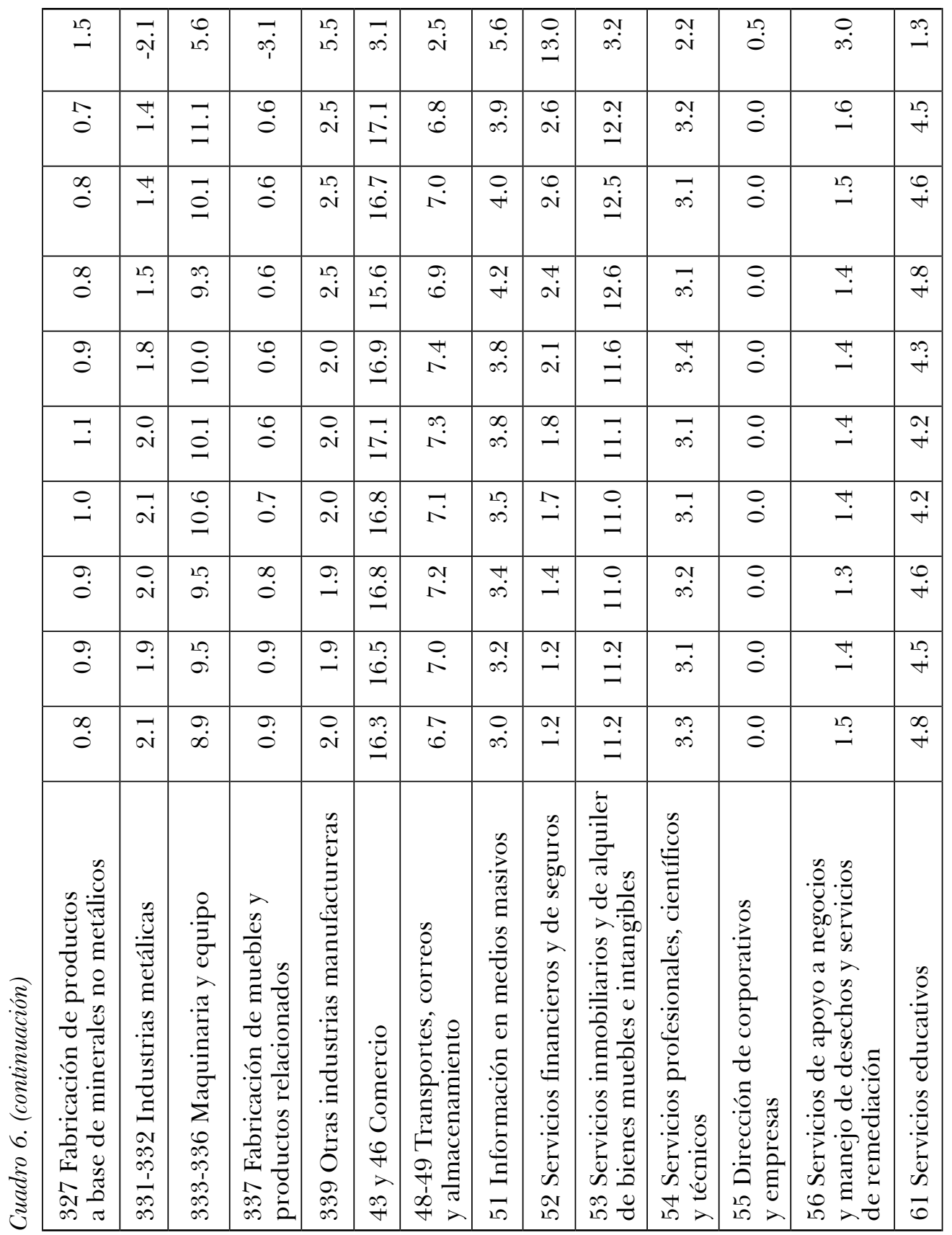




\begin{tabular}{|c|c|c|c|c|c|}
\hline$\dot{0}$ & $\underset{\text { or }}{\overrightarrow{0}}$ & بִ & 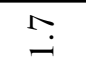 & $\stackrel{\nabla}{\sim}$ & $\stackrel{0}{\dot{\theta}}$ \\
\hline $\overrightarrow{o r}$ & $\stackrel{0}{0}$ & o? & $\vec{o}$ & $\stackrel{0}{+}$ & ִִּ \\
\hline $\begin{array}{l}\text { ov } \\
\text { on }\end{array}$ & $\stackrel{0}{0}$ & $\overrightarrow{0}$ & $\begin{array}{l}\infty \\
\infty \\
\sim\end{array}$ & Oִ & $\stackrel{\not}{\sim}$ \\
\hline $\begin{array}{l}\text { की } \\
\text { of }\end{array}$ & $\stackrel{0}{0}$ & $\begin{array}{l}\text { ọ } \\
\text { or }\end{array}$ & $\begin{array}{l}\text { O? } \\
\text { ov }\end{array}$ & $\stackrel{\infty}{+\infty}$ & $\stackrel{+}{\sim}$ \\
\hline$\underset{\text { of }}{0}$ & ?ִ & $\begin{array}{l}\text { ọ } \\
\text { or }\end{array}$ & $\hat{\sigma}$ & or & ִִ \\
\hline $\begin{array}{l}\text { of } \\
\text { of }\end{array}$ & ?ִ & or & $\begin{array}{l}0 \\
\text { ov }\end{array}$ & $\begin{array}{l}\infty \\
\infty\end{array}$ & $\stackrel{\longrightarrow}{-}$ \\
\hline $\begin{array}{l}0 \\
\text { os }\end{array}$ & $\stackrel{20}{0}$ & $\begin{array}{l}\text { of } \\
\text { of }\end{array}$ & $\begin{array}{l}0 \\
\text { ov }\end{array}$ & or & $\ddot{0}$ \\
\hline or & ?ִ & $\underset{+}{7}$ & a & $\begin{array}{l}\text { o? } \\
\text { or }\end{array}$ & $\dot{0}$ \\
\hline $\begin{array}{l}\infty \\
\infty \\
\infty\end{array}$ & ?ִ & $\begin{array}{l}\text { o? } \\
\text { or }\end{array}$ & $\hat{\sigma}$ & $\begin{array}{l}\text { o? } \\
\text { or }\end{array}$ & $\ddot{0}$ \\
\hline $\begin{array}{l}0 \\
\text { of }\end{array}$ & ?ִ & تُ & $\begin{array}{l}0 \\
\text { Oे }\end{array}$ & Oִ & $\ddot{0}$ \\
\hline 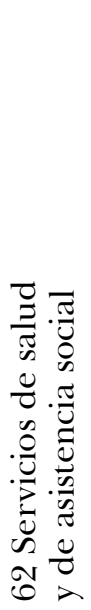 & 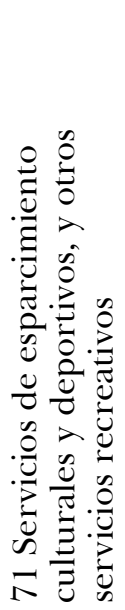 & 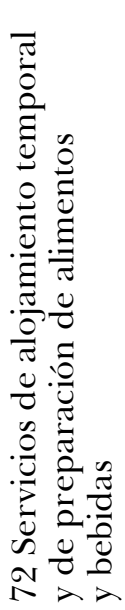 & 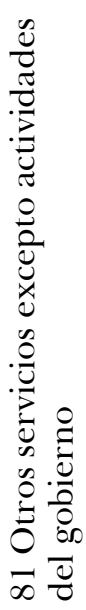 & 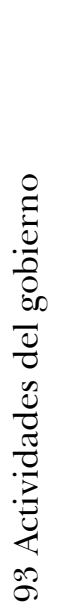 & 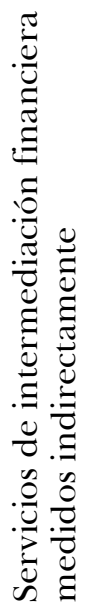 \\
\hline
\end{tabular}


cada uno de ellos propician actividades empresariales que las aprovechan. Los municipios de Ensenada y Rosarito, caracterizados por su vocación hacia el turismo, tienen un mayor porcentaje de población ocupada en actividades relacionadas con el comercio al por menor y servicios de alojamiento temporal, con una participación de $34.8 \%$ y $36.6 \%$ respectivamente. Destaca que Ensenada tenga $70 \%$ de la población ocupada en el sector primario, principalmente en agricultura de exportación, producción vitivinícola y explotación de recursos pesqueros. Por su parte, Tecate, Tijuana y Mexicali tienen actividades preponderantemente manufactureras, con una participación de la población ocupada en dicho sector de $48.8 \%$, $34.7 \%$ y $41.6 \%$ respectivamente. Sin embargo, al analizar las economías municipales por el número de establecimientos, destaca la mayoritaria participación de los dedicados al comercio al por menor, los cuales representan $45.4 \%$ en Tecate, $44.3 \%$ en Rosarito, $42.8 \%$ en Tijuana, $42.1 \%$ en Ensenada y $37.5 \%$ en Mexicali.

\section{Estructura industrial y empleo por tamaño de empresa}

La importancia de Baja California como sitio de localización para las empresas maquiladoras norteamericanas y asiáticas de alto nivel tecnológico es tal, que con fines comparativos, la entidad cuenta con entre 18 y $19 \%$ de las plantas maquiladoras del país y entre 11 y $12 \%$ de los empleos, y alrededor de $18 \%$ del valor agregado maquilador generado en México. El impacto de la crisis se ha notado en el descenso de establecimientos industriales y en el más lento crecimiento del empleo, no obstante que la maquiladora de Baja California se ubica en el primero y segundo lugar a escala nacional respectivamente, en ambos indicadores. Esto es ilustrativo del impacto regional del proceso de modernización tecnológica que este tipo de plantas están viviendo a escala mundial, pues a la vez que generan un mayor crecimiento de la productividad, están generando un menor crecimiento del empleo (Rotman, 2013) (cuadro 7).

Baja California se caracteriza por tener un crecimiento industrial fuertemente basado en la subcontratación que se origina en la complementación industrial regional, tanto moderna de alta tecnología asociada a electrónica, aeroespacial y productos médicos, como tradicional vinculada 


\section{Cuadro 7. Indicadores de la industria maquiladora} de exportación, 1990-2012

\begin{tabular}{|c|c|c|c|c|c|c|c|}
\hline \multirow{2}{*}{ Año } & & \multicolumn{2}{|c|}{ Establecimientos } & \multicolumn{2}{|c|}{ Personal Ocupado } & \multicolumn{2}{|c|}{$\begin{array}{l}\text { Valor agregado } \\
\text { (Miles de pesos) }\end{array}$} \\
\hline & & Número & $T C$ & Número & $T C$ & Número & $T C$ \\
\hline \multirow{3}{*}{1990} & $\begin{array}{l}\text { Baja } \\
\text { California }\end{array}$ & 669 & & 84573 & & 2011069 & \\
\hline & Nacional & 1789 & & 937183 & & 9918504 & \\
\hline & $\begin{array}{l}\text { Frontera } \\
\text { Norte }\end{array}$ & 1595 & & 407744 & & 9093631 & \\
\hline \multirow{3}{*}{2000} & $\begin{array}{l}\text { Baja } \\
\text { California }\end{array}$ & 1279 & 6.5 & 282602 & 12.1 & 36102883 & 28.9 \\
\hline & Nacional & 3703 & 7.3 & 1310026 & 3.4 & 163414471 & 28.0 \\
\hline & $\begin{array}{l}\text { Frontera } \\
\text { Norte }\end{array}$ & 2846 & 5.8 & 1083740 & 9.8 & 130899521 & 26.7 \\
\hline \multirow{3}{*}{2009} & $\begin{array}{l}\text { Baja } \\
\text { California }\end{array}$ & 988 & -2.9 & 203831 & -3.6 & 51420916 & 3.5 \\
\hline & Nacional & 5245 & 3.9 & 1641465 & 2.5 & 291079261 & 5.8 \\
\hline & $\begin{array}{l}\text { Frontera } \\
\text { Norte }\end{array}$ & 3167 & 1.2 & 998992 & -0.9 & 225415485 & 5.4 \\
\hline \multirow{3}{*}{2012} & $\begin{array}{l}\text { Baja } \\
\text { California }\end{array}$ & 898 & -3.2 & 230396 & 4.1 & n.d & n.d \\
\hline & Nacional & 5049 & -1.3 & 1989862 & 6,4 & n.d & n.d \\
\hline & $\begin{array}{l}\text { Frontera } \\
\text { Norte }\end{array}$ & 3042 & -1.3 & 1215236 & 6.5 & n.d & n.d \\
\hline
\end{tabular}

Fuente: INEGI. 
con textiles y plásticos. La fuerte presencia y concentración de plantas de origen asiático en el corredor Tijuana-Mexicali, con 30\% de la inversión maquiladora de la zona, ha convertido a la entidad en el centro productor de televisores más grande del mundo. En consecuencia, $90 \%$ de las empresas generadoras de los contratos de las empresas maquiladoras en Baja California, y un buen número de proveedores de insumos industriales, se ubican en el corredor Los Ángeles-San Diego. Hay que señalar que si bien Baja California es de los estados fronterizos más beneficiados con las prácticas de industrialización orientadas a la exportación bajo diversas modalidades de subcontratación, también es claro que a diferencia de otros estados del norte, como Nuevo León y Chihuahua, su principal limitante ha sido el desarrollo de redes de proveedores. Esta situación ha llevado a que mientras el saldo neto de inversión extranjera directa ha crecido en estos estados de $10.3 \%$ a $11.6 \%$ y de $3.5 \%$ a $6.5 \%$ respectivamente, Baja California y el resto de los estados fronterizos apenas han crecido en sus saldos (cuadro 8).

La intensa presencia de la maquiladora durante la década de 1960, motivada por la amplia disponibilidad de fuerza de trabajo y la expedición de reglas federalmente establecidas que protegen su operación, da

Cuadro 8. Saldo de inversión extranjera directa por entidad federativa fronteriza y participación en total nacional, 2001-2010

\begin{tabular}{|c|r|r|r|r|}
\hline Entidad federativa & \multicolumn{1}{|c|}{$2001-2005$} & \multicolumn{1}{c|}{$\%$} & \multicolumn{1}{c|}{$2006-2010$} & \multicolumn{1}{c|}{$\%$} \\
\hline Baja California & 4722.7 & 3.9 & 4800.6 & 4.0 \\
\hline Coahuila & 4233.3 & 3.5 & 7752.5 & 6.5 \\
\hline Chihuahua & 895.4 & 0.7 & 1749.6 & 1.5 \\
\hline Nuevo León & 12536.9 & 10.3 & 13777.9 & 11.6 \\
\hline Sonora & 1076.0 & 0.9 & 2474.3 & 2.1 \\
\hline Tamaulipas & 1639.4 & 1.3 & 1960.2 & 1.7 \\
\hline
\end{tabular}

Fuente: Secretaría de Economía. 
lugar a la joven vocación industrial que se amplía con el intenso proceso de promoción industrial realizado durante la década de 1980 a partir de una política de incentivos fiscales a las empresas que se instalaran en la zona. Entre los años de 1990 y la primera década del siglo xxı, al amparo de los programas federales de promoción industrial que privilegian las actividades de alta rentabilidad a través de la promoción de exportaciones, las actividades de maquila se apoderan de la atención de los gestores y promotores del desarrollo industrial (Mungaray, Ramos, Placencia y Moctezuma, 2011).

Las experiencias asiáticas han mostrado que una base nacional de exportación puede desarrollarse con más facilidad en un ambiente económico de apertura con protección discriminada, que en uno de protección indiscriminada (Ohmae, 1995). La industrialización orientada a la exportación con un sustento en el aprendizaje y la tecnología, ha sido creciente en Baja California desde mediados de los años de 1980. Ello ha abierto claras posibilidades para integrar una política de desarrollo científico y tecnológico a la estrategia económica de la región, como parte de una política industrial orientada a construir ventajas competitivas para la nación.

Frente a esta problemática, las relaciones entre los promotores de inversión y el gobierno del estado generaron estrategias efectivas de integración y coordinación para superar las fallas de mercado que impiden la formación y el desarrollo más amplio del pequeño empresario nacional. El hecho de que las instituciones educativas realicen actividades de capacitación que no encuentran mercado y que las empresas requieran servicios de capacitación que no pueden ser satisfechas por éstas, o el hecho de que las grandes y estables empresas maquiladoras tengan que importar la casi totalidad de sus insumos mientras un buen número de pequeñas empresas nacionales sufren la falta de mercados para sus productos, son ejemplos que dan idea de la insuficiencia de mecanismos institucionales coordinadores de los agentes económicos. Esto genera una reorientación de la oferta de educación superior del estado hacia la formación de recursos humanos con capacidad técnica y general, para ser incorporados en actividades productivas orientadas tanto al mercado local como mundial (Moctezuma, Ocegueda, Mungaray; Ocegueda y Estrella, 2013). A la vez, son un indicador de la posibilidad de convertir las fallas de mercado en 
oportunidades de desarrollo empresarial, aprendizaje tecnológico y generación de mayor valor agregado.

Por su parte, los intensos cambios en la política comercial nacional desde mediados de la década de 1980 y los negativos efectos sobre la estructura comercial de la frontera han cambiado el peso del sector dentro de la estructura del empleo. Para 1990, el proceso de apertura y los cambios legislativos e institucionales que lo sustentan conducen a que el peso de la industrializacion vía maquiladoras y el ascenso del espíritu empresarial se traduzcan en un importante aumento de establecimientos industriales, principalmente a nivel de las micro y pequeñas empresas y del número de empleos creados por ellas.

De acuerdo con los censos económicos, el estado contaba con 59586 empresas en 2003 y 80654 en 2008, lo cual indica un crecimiento en 21068 establecimientos. Al descomponer estas cifras según estrato empresarial, puede notarse que los incrementos más importantes fueron en el estrato de micro y pequeñas empresas en 18863 y 2042 establecimientos respectivamente. En general, la aportación de las microempresas en la ocupación estatal se ha mantenido entre $43 \%$ y $41.1 \%$ entre 2005 y 2012 ; y junto con la pequeña empresa que permanece en el orden del $25 \%$, aportan actualmente $66.6 \%$ del empleo total.

El estrato de las empresas compuesto por las medianas y grandes aporta el resto del empleo y su contribución va a menos debido a la pérdida de empleo de la mediana empresa, que entre 2005 y 2012 pasa de $20.5 \%$ a $18.3 \%$. La gran empresa se encuentra en una fase de pérdida o lento crecimiento del empleo, pues mientras que entre 2006 y 2007 se mantiene en 19\% del empleo total, para 2012 baja a 15\%. Esta situación se asocia, primero, a una disminución temporal de contratos en el mercado mundial debido a la crisis económica internacional y, luego, a la necesaria automatización de sus procesos productivos para mantenerse competitiva en términos de producción de mayor valor agregado por empleo generado (cuadro 9).

En la Baja California de 2012, sin duda los estratos que más aportan a la creación de empleo son los micro y pequeños negocios, pues mientras que durante 2005-2012 se crean más de 180 mil empleos en el estado, las micro y pequeñas empresas aportaron más de 55 mil y 83 mil, respectivamente, muy por encima de las grandes, que no llegan a $30 \mathrm{mil}$, y las medianas, que aportaron menos de 12 mil empleos (cuadro 10). 


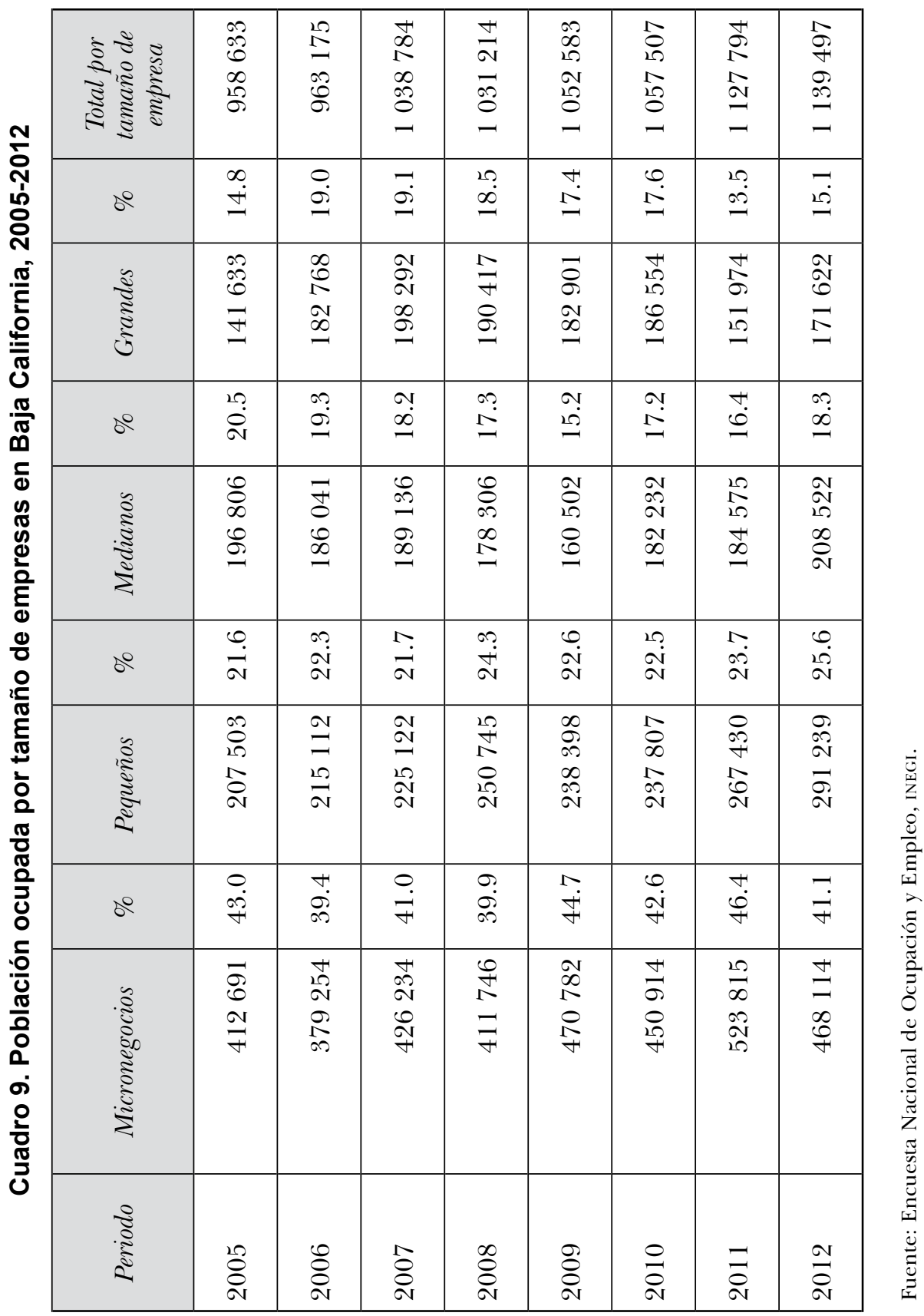




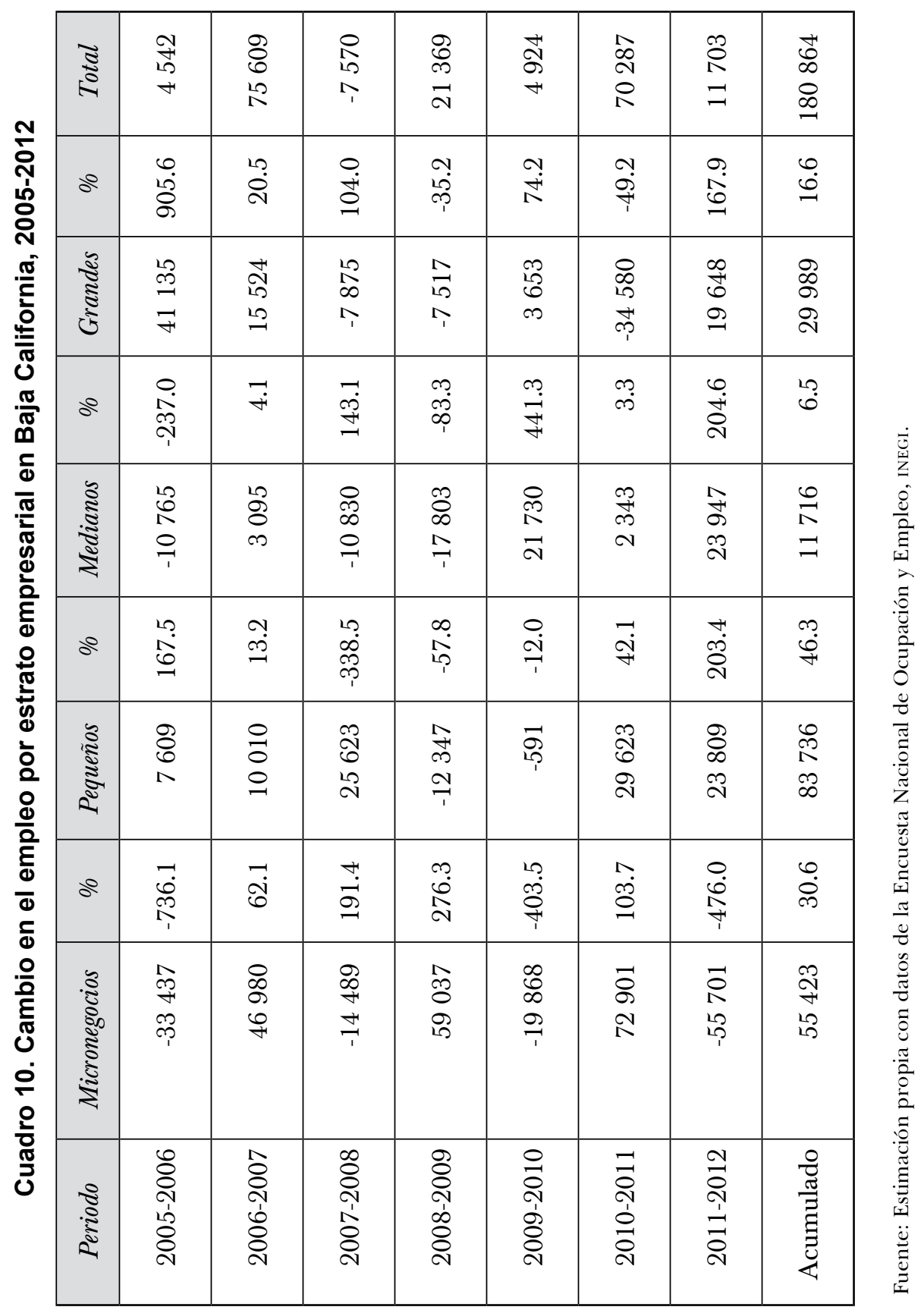


Los datos anteriores reflejan que si bien la inversión extranjera y la industria maquiladora de exportación en términos de su aportación al empleo manufacturero ha sido importante, la dinámica que en los últimos años ha mostrado el estrato de mypes se apuntala como la principal fuente de creación de empleo hacia la primera década de este siglo, pues por sí mismas produjeron más de 139 mil de los 159 mil empleos creados (cuadro 11).

Cuadro 11. Población ocupada por tamaño de empresa y sector de actividad, 2005-2012

\begin{tabular}{|c|c|c|c|c|c|c|c|}
\hline \multirow{2}{*}{ Año } & \multirow{2}{*}{$\begin{array}{l}\text { Tipo de unidad } \\
\text { económica }\end{array}$} & \multicolumn{2}{|c|}{$\begin{array}{c}\text { Industrias } \\
\text { manufactureras }\end{array}$} & \multicolumn{2}{|c|}{ Comercio } & \multicolumn{2}{|c|}{ Servicios } \\
\hline & & $\begin{array}{l}\text { Personal } \\
\text { ocupado }\end{array}$ & $\%$ & $\begin{array}{l}\text { Personal } \\
\text { ocupado }\end{array}$ & $\%$ & $\begin{array}{l}\text { Personal } \\
\text { ocupado }\end{array}$ & $\%$ \\
\hline \multirow{6}{*}{2005} & Total & 255771 & 100 & 224999 & 100 & 425353 & 100 \\
\hline & Micronegocios & 41161 & 16.1 & 120034 & 53.3 & 164451 & 38.7 \\
\hline & $\begin{array}{l}\text { Pequeños } \\
\text { establecimientos }\end{array}$ & 13529 & 5.3 & 32152 & 14.3 & 149065 & 35.0 \\
\hline & $\begin{array}{l}\text { Medianos } \\
\text { establecimientos }\end{array}$ & 95191 & 37.2 & 60293 & 26.8 & 30613 & 7.2 \\
\hline & $\begin{array}{l}\text { Grandes } \\
\text { establecimientos }\end{array}$ & 105890 & 41.4 & 12520 & 5.6 & 16689 & 3.9 \\
\hline & Otros & & & & & 64535 & 15.2 \\
\hline \multirow{6}{*}{2012} & Total & 262450 & 100 & 293305 & 100 & 586768 & 100 \\
\hline & Micronegocios & 50615 & 19.3 & 155280 & 52.9 & 202365 & 34.5 \\
\hline & $\begin{array}{l}\text { Pequeños } \\
\text { establecimientos }\end{array}$ & 16036 & 6.1 & 42047 & 14.3 & 215448 & 36.7 \\
\hline & $\begin{array}{l}\text { Medianos } \\
\text { establecimientos }\end{array}$ & 61270 & 23.3 & 90517 & 30.9 & 49727 & 8.5 \\
\hline & $\begin{array}{l}\text { Grandes } \\
\text { establecimientos }\end{array}$ & 134529 & 51.3 & 5461 & 1.9 & 24067 & 4.1 \\
\hline & Otros & & & & & 95161 & 16.2 \\
\hline
\end{tabular}

Fuente: Encuesta Naciona de Ocupación y Empleo, INEGI. 
En consistencia con el desempeño de la producción de las manufacturas, la población ocupada en los micronegocios mostró un aumento en su participación en este sector, al pasar de $16.1 \%$ en las manufacturas, a $19.3 \%$ entre 2005 y 2012. En los micronegocios de los sectores del comercio y los servicios, por su parte, no obstante el aumento en su población ocupada en términos absolutos, en términos relativos se observó una disminución de $53.3 \%$ a $52.9 \%$ el primero, y de $38.7 \%$ a $34.5 \%$ el segundo, entre 2005 y 2012.

\section{Conclusiones}

A pesar de transitar en un difícil momento coyuntural, abierto por una crisis económica mundial, la evolución de los indicadores de población, producción y ocupación de Baja California mantienen la tendencia de crecimiento a largo plazo. Esto consolida la posición del estado como un polo de atracción de empresas y de corrientes migratorias.

Baja California se mantiene dentro de los estados con mayor captación de inversión extranjera en el país. En 2012 se ubica en el sexto lugar nacional y segundo entre los estados de la frontera norte, con un aumento en las ventas al por menor de $4.5 \%$ por arriba del promedio nacional de 4.3\%; la producción manufacturera muestra una clara tendencia a la recuperación y en octubre de 2012 alcanza su nivel histórico más alto; el valor de la construcción total ha alcanzado su mayor crecimiento en noviembre de 2012 frente al obtenido en 2009, y el segundo mayor a nivel histórico; el valor de la construcción pública se ha incrementado en $14.4 \%$ en el periodo de enero a noviembre de 2012 por el fuerte impulso del gasto público en el sector; y la recepción de remesas tuvo el mayor impacto entre las entidades del país, con un aumento de 17.2\% durante 2012.

La tarea que sigue pendiente es articular los procesos de producción de la industria maquiladora con actividades industriales locales que sustenten dinámicas de desarrollo industrial de largo plazo que las actividades tradicionales de maquila no han profundizado en apoyo a la formación empresarial de la zona. Hoy no se puede negar que la maquiladora ha contribuido a desarrollar el crecimiento industrial en el estado y que ha influido de manera importante en la conformación de una nueva base regional 
de crecimiento económico, que es base de la transformación de todos los aspectos de la dinámica tradicional de la región. Sin embargo, la escasa integración de insumos regionales a las maquiladoras de exportación refleja problemas de información e integración, pero también un área de oportunidad en la estrategia competitiva de la industria local y nacional.

Asimismo, debido a la importancia estratégica de las micro y pequeñas empresas como generadoras de ocupación, se debe fortalecer la política industrial que favorece el aprendizaje y la innovación empresarial como elementos esenciales que les permitan crecer y competir en los mercados internacionales cada vez más competitivos, ya sea directamente o como proveedores en la cadena de valor de las grandes empresas de exportación. Son estas micro y pequeñas empresas las que están absorviendo el desempleo generado por las grandes empresas internacionales y/o el flujo migratorio que busca oportunidades laborales que no encuentra en sus lugares de origen.

La composición de la estructura económica y del empleo de Baja California permitió que la disminución de la producción en el sector secundario (manufacturas y construcción) fuese compensada por el crecimiento de los sectores primario y comercial, que se vio impactado favorablemente por el tipo de cambio. Este efecto compensatorio, aunado al repunte de los principales indicadores de los más importantes socios comerciales, mantiene prudentes expectativas de recuperación económica en corto y mediano plazos y una tendencia de crecimiento de largo plazo.

El desarrollo regional es un proceso de cambio que implica tanto el crecimiento económico como el mejoramiento de las condiciones de vida de la población. Por ello, una política industrial orientada a incentivar el desarrollo regional a través de la infraestrucutra física y educativa favorece la empresarialidad y el desarrollo de redes de proveedores como parte fundamental de los encadenamientos industriales a partir de las relaciones empresariales existentes (Bertelli y Moye, 1997; Humphrey y Schmitz, 2002).

Para tal fin, la actual política de desarrollo empresarial debe mantener la orientación de la estructura industrial, comercial y de servicios del Estado hacia la producción de bienes y servicios con mayor valor agregado; articular el entorno regional de competitividad para maximizar los beneficios de la inversión nacional y extranjera en la formación y acumulación de innovación a través de cadenas de valor con las micro, pequeñas 
y medianas empresas; e impulsar una economía del conocimiento, altamente competitiva y socialmente incluyente.

\section{Bibliografía}

Banco de México. (2013). Encuesta sobre las Expectativas de los Especialistas en Economía del Sector Privado. Recuperado de < http://www.banxico.org.mx/informacionpara-la-prensa/comunicados/resultados-de-encuestas/expectativas-de-losespecialistas/index.html>.

Banco de México. (2013). Ingresos por remesas familiares, distribución por entidad federativa. Recuperado de <http://www.banxico.org.mx/SieInternet/consulta rDirectorioInternetAction.do?accion $=$ consultarCuadro\&idCuadro $=\mathrm{CE} 100 \&$ sector $=1 \&$ locale $=\mathrm{es}>$.

Banco Mundial. (2013). The global outlook in summary. Recuperado de $<$ http:// web.worldbank.org/wBSITE/EXTERNAL/EXTDEC/EXTDECPROSPECTS/EXTGBLPROSPECT /0, contentмDк:23327491 menuрк:612510 pagepк:2904583 рігк:2904598 $\sim$ theSitepк:612501,00.html>.

Bertelli, D. y Moye, M. (1997). Un nuevo enfoque para la política industrial a nivel regional. En E. Dussel, M. Piore y C. Ruiz, Pensar globalmente y actuar regionalmente. Hacia un nuevo paradigma industrial para el siglo XXI (pp. 389-433). México: Jus, Fes-unAm.

Bureau of Transportation and Statistics. (2013). North American transborder freight data. Recuperado de <http://transborder.bts.gov/programs/international/transborder/TBDR_QA.ht ml $>$.

Bureau of Transportation and Statistics. (2013). Border crossing. Recuperado de $<$ http://transborder.bts.gov/programs/international/transborder/TBDR_BC/ TBDR_BC_Index.html >.

Conapo (Consejo Nacional de Población). (2010). Proyecciones de la población en México 2005-2050. Recuperado de www.conapo.gob.mx.

DOF (Diario Oficial de la Federación). (23 de enero de 2012). Decreto de competitividad y reducción arancelaria de la zona económica fronteriza.

Escamilla, A. y Ocegueda, J. M. (2011). Volatilidad del crecimiento económico y patrones de especialización óptima en la frontera norte de México. México: Universidad Autónoma de Baja California, Porrúa.

Estrella, G. (1998). Perfil de la población urbana en la frontera norte de México. Comercio Exterior, 48(5), 378-383.

Fondo Monetario Internacional. (2012). Perspectivas de la economía mundial. Recuperado de <http://www.imf.org/external/spanish/pubs/ft/weo/2012/02/ pdf/texts.pdf $>$. 
Humphrey, J. y Schmitz, H. (2002). How does insertion in global value chains affect upgrading in industrial clusters? Regional Studies, 9, 1017-1027.

Instituto Mexicano para la Competitividad. (2008). Competitividad estatal de México, 2008. Aspiraciones y realidades: Las agendas del futuro. México: Autor.

Instituto Nacional de Estadística y Geografía. (2013). Encuesta Nacional de Ocupación y Empleo. Recuperado de <http://www.inegi.org.mx/Sistemas/ infoenoe/TriPreliminar.aspx?s $=$ est $\& c=27736 \& \mathrm{p}=>$.

Instituto Nacional de Estadística y Geografía. (2013). Censos económicos. Recuperado de < http://www.inegi.org.mx/est/contenidos/Proyectos/ceDefault. aspx $>$.

Instituto Nacional de Estadística y Geografía. (2013). Construcción encuesta mensual. Recuperado de < http://www.inegi.org.mx/sistemas/bie/>.

Instituto Nacional de Estadística y Geografía. (2013). Producción manufacturera por entidad federativa. Recuperado de <http://www.inegi.org.mx/sistemas/bie/ > .

Instituto Nacional de Estadística y Geografía. (2013). Producto interno bruto por entidad federativa. Recuperado de < http://www.inegi.org.mx/sistemas/bie/> .

Instituto Nacional de Estadística y Geografía. (2013). Industria manufacturera, maquiladora y de servicios de exportación. Recuperado de < http://www.inegi.org. $\mathrm{mx} / \mathrm{sistemas} / \mathrm{bie} />$.

Mendoza, J. E. (2010). El comportamiento de la industria manufacturera de México ante la recesión económica de Estados Unidos de América. Revista de Economía, XXVII(75), 9-35.

Moctezuma, P., Ocegueda, M. T., Mungaray, A., Ocegueda, J. M. y Estrella, G. (2013). Cobertura con calidad a través de la reorganización institucional en la educación superior de Baja California. Frontera Norte, 25(49), 87-108.

Mungaray A. y Moctezuma, P. (1988). El mercado de la frontera norte de México y las políticas de integración del consumo fronterizo a la producción nacional. Revista Mexicana de Sociología, L(4), 227-244.

Mungaray, A. y Ocegueda J. M. (1995). La nueva frontera norte: Entre la devaluación y la 187. Comercio Exterior, 45(6), 450-459.

Mungaray, A., Ramos, J., Plascencia, I. y Moctezuma, P. (2011). Las instituciones de educación superior en el sistema regional de innovación de Baja California. Revista de la Educación Superior, xL(2), 158,119-136.

Mungaray, A. y Samaniego, M. (2006). De 1945 a nuestros días. Internacionalización económica y democracia política en Baja California. En M. A. Samaniego, Breve historia de Baja California (pp. 183-229). México: Miguel Ángel Porrúa, Universidad Autónoma de Baja California.

Ohmae, K. (1995). The end of the nation state. The rise of regional economics Londres: Harper Collins PbS.

Rotman, D. (2013). How technology is destroying jobs. Technology Review, 16(4), 28-35. 
San Diego Regional Planning Agency. (2008). 2030 Regional growth forecast update. Recuperado de www.sandag.org.

Secretaría de Economía. (2013). Estadística oficial de los flujos de IED hacia México Recuperado de <http://www.economia.gob.mx/files/comunidad_negocios/ied/ informe_congreso_4t_2012.pdf $>$. 